\title{
Current characteristics of a one-dimensional Hubbard chain: The role of correlation and dissipation
}

\author{
Jakob Neumayer ${ }^{*}$ Enrico Arrigoni, Markus Aichhorn, and Wolfgang von der Linden \\ Institute of Theoretical and Computational Physics, \\ Graz University of Technology, NAWI Graz, 8010 Graz, Austria
}

(Dated: October 12, 2018)

\begin{abstract}
We study the electronic transport in an infinite one-dimensional Hubbard chain, driven by a homogeneous electric field. The physical chain is coupled to fermion bath chains, in order to account for dissipation and to prevent the occurrence of Bloch Oscillations. The steady state current is computed in the frame of Keldysh Green's functions in Cluster Perturbation Theory. The current characteristics are dominated by resonant-tunneling-like structures, which can be traced back to Wannier-Stark resonances due to anti-ferromagnetic correlations. The same current characteristic occurs in a non-interacting Wannier-Stark model with alternating on-site energies. Non-local effects of the self energy can be accounted for the observed physical behaviour.

PACS numbers: 71.15.-m, 71.27+a, 71.10. -w, 73.23.-b
\end{abstract}

\section{INTRODUCTION}

One dimensional (1d) quantum systems exhibit fascinating non-equilibrium effects such as Bloch oscillations $^{10}$ (BOs), Zener tunneling ${ }^{2}$ and Wannier-Stark resonances. ${ }^{3}[5]$ The first theoretical studies of these effects date back to the early days of condensed matter physics and were followed by long-lasting controversial discussions about their relevance in real materials. ${ }^{6}[8$ It was only within the last two decades that the theoretical predictions could be observed in condensed matter sytems and optical potentials in a series of ingenious experiments.9.19. A detailed overview over the most important experimental verifications of the above mentioned effects is provided in Ref. 20 and Ref. 21, where also experimental set-ups for determining Wannier-Stark resonances with cold atoms in optical lattices are introduced.

Bloch oscillations are mainly studied in 1d systems which are and were frequently used as model systems to investigate the physical nature of BOs. As far as correlations are concerned the 1d Hubbard model was mostly the model of choice. The clear evidence for the existence of BOs and Wannier-Stark resonances led to a series of further theoretical investigations of $1 \mathrm{~d}$ structures under the impact of a uniform force induced by an electric or magnetic field! $\frac{17|20| 22 \mid 25}{2}$

For the emergence of Wannier-Stark resonances in $1 \mathrm{~d}$ systems a periodic potential has to be applied. It was long thought that this periodic potential has to meet some specific conditions ${ }^{[26]}$ Recently, it was shown analytically that any periodic potential suffices to cause these resonant quantum states ${ }^{33}$

The theoretical investigation of non-equilibrium properties have become very popular in recent years stimulated by the experimental progress and based on the development of powerful numerical methods that allow to tackle these challenging problems. The nonequilibrium characteristics of $1 \mathrm{~d}$ systems in a homogeneous electrical field, resulting in a linearly decreasing potential, have been studied with various theoretical methods and in different geometries and gauges. One route of research considers $1 \mathrm{~d}$ rings in the so-called temporal gauge, i.e. the ring is threaded by a magnetic flux that increases linearly in time. The advantage of this geometry is that one can use periodic boundary conditions. For non-interacting particles the problem can be solved analytically for arbitrary ring size in the Keldysh formalism. For a review see Ref. 24. It has been found that there is no dc-current possible without additional dissipation channels. The latter can be introduced by attaching an infinite fermionic bath chain to each site of the ring. As soon as electron-electron interactions are included, the problem can only be solved by numerical means and for very small ring sizes without dissipative bath chains. The real-time evolution for a 10-site ring has been studied ${ }^{22}$ for Hubbard interaction by the Cranck-Nicholson method and in Ref. 34 for the extended Hubbard model by the Chebyshev propagation method. In both cases no steady state dc current has been found, due to the lack of dissipation processes. In Ref. 34 it was shown that the current characteristics are always dominated by BOs.

Another class of studies considers a finite 1d Hubbard chain (central region) with linearly decreasing onsite energies, which are attached on both ends to leads, represented by finite tight-binding (TB) chains of noninteracting electrons. Time-dependent density-matrix renormalization group (tDMRG) calculations have been performed for a central region of 10 sites attached to 20 lead sites on both ends. $\frac{35}{5}$ The results are in qualitative agreement with those found for the closed ring geometry, ${ }^{[2]}$ with the quintessence of an universal dielectric breakdown characteristic of the Mott insulating state. It is worth mentioning that a similar result was found by dynamical mean-field theory (DMFT) calculations on the hyper-cubic lattice ${ }^{36}$ However, in all realtime evolutions the current did not reach the steadystate.

Yet another possible setup to study the response of one dimensional lattice fermions to a homogeneous electric 
field is an infinite 1d TB-chain with linearly decreasing on-site energies with or without electron-electron interactions. In the non-interacting case the temporal gauge has been studied in great detail in Ref. 37, where a steady state was obtained by attaching fermionic dissipative baths to each site of the chain. The dissipation carries away the extra energy accumulated and is essential to suppress BOs and to obtain a finite steady state current. Also in the two-dimensional one-band Hubbard mode ${ }^{38}$ an explicit coupling to fermion bath chains was concluded to be essential for obtaining a description of non-equilibrium steady-states. It should be noted that in set-ups where a finite physical device is attached to infinite leads, dissipation is already provided by the leads. 39 41

Clearly, fermionic heat baths appear rather artificial at first glance. However, a closer analysis reveals that their impact on a physical system is qualitatively the same as that of phonons $\frac{37}{37}$ Recent quantum many-body studies on this topic showed that many physical properties in model systems with fermion baths behave as predicted by the classical Boltzmann transport theory $[37 / 42[43]$ Further arguments in favour of fermionic baths can be found in Refs. 43 or 44, where the non-equilibrium steady-state of photo-excited correlated electrons has been studied. The fermion bath model was also used earlier to describe the electron transport in a metallic ring ${ }^{45}$ where it was denoted as an extended Büttiker's model, It has to be mentioned here, that in some systems dissipation mechanisms do not have to be included explicitly since they are included anyways by means of the setup.

In the present work, we will study an infinite Hubbardchain in a homogeneous electric field. The dissipation will be described by fermionic baths ${ }^{37 / 38 / 46}$ attached to each site of the physical chain. This approach is particularly convenient from the theoretical point of view. In contrast to previous works the non-equilibrium steady state of the interacting many-body system is treated by a generalization of Cluster Perturbation Theory (CPT) ${ }^{4748}$ to the non-equilibrium situation. $\frac{40 / 41}{4}$ This allows to study the impact of dissipation and electron-electron interactions on the transport of lattice fermions in an infinite homogeneous electric field. Furthermore, non-local effects of the self energy can be included via CPT. It will be shown later, that the inclusion of those non-local effects is crucial to explain the resonant structures occurring in the current characteristics of the correlated Hubbard chain.

The paper is organized as follows: In Sec. II we give an introduction to the model and its solution. The current characteristics in the non-interacting case are briefly summarized in Sec. III A to allow for a comprehensive understanding of the current characteristics expected in infinite 1d structures. The effect of on-site Coulomb interactions is discussed subsequently in Sec. IIIB To gain a comprehensible physical explanation of the results, we describe an alternative way of modelling a noninteracting system with a similar non-equilibrium behaviour as the interacting one in Sec. IIIC. We will close

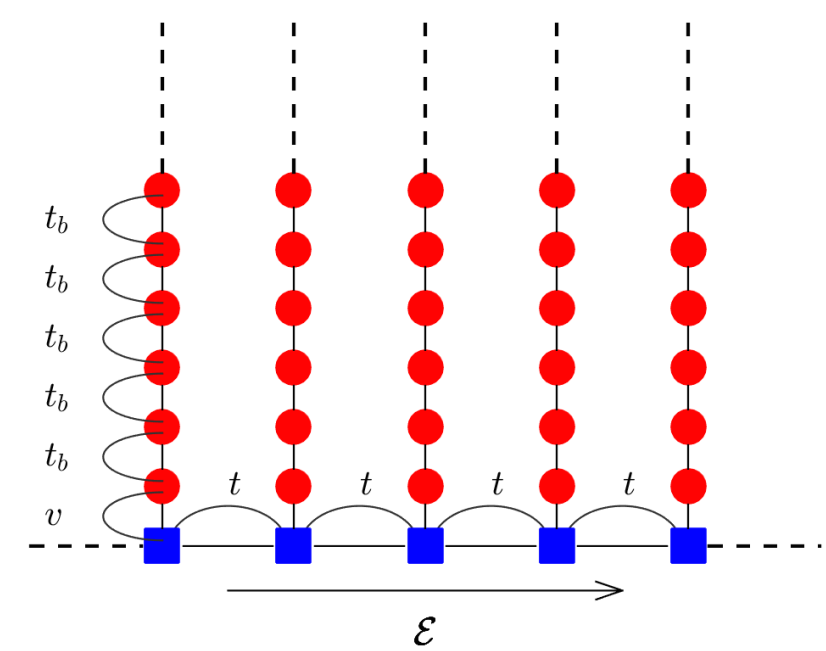

FIG. 1: (Color online) Sketch of the infinite one-dimensional correlated chain (blue squares), coupled at each site to individual 1d heat bath chains (red circles). The homogeneous electric field $\mathcal{E}$ is applied parallel to the physical chain.

our considerations by a simplified nonequilibrium Variational Cluster Approach (VCA) treatment in Sec. IIID. The final conclusion is presented in Sec. IV.

\section{THEORETICAL TREATMENT}

The setup of the model is depicted in Fig.1. Blue squares represent the correlated physical sites of the 1D Hubbard chain. Each physical site is coupled via hopping to fermionic bath chains. The parameters $t, t_{b}, v$, as depicted in the figure, stand for the hopping along the physical TB chain, within the bath chains, and the hopping between the physical system and the heat bath chains. The uniform electric field is applied parallel to the physical chain.

\section{A. The Hubbard-Wannier-Stark model}

The Hamiltonian corresponding to the system depicted in Fig. 1 is reads

$$
\mathcal{H}=\mathcal{H}_{0}+\mathcal{H}_{\mathrm{U}}+\mathcal{H}_{\text {bath }} .
$$

The kinetic part of the correlated chain is described by a TB-Hamiltonian

$$
\mathcal{H}_{0}=-t \sum_{\langle i, j\rangle, \sigma} c_{i \sigma}^{\dagger} c_{j \sigma}+\sum_{j} \epsilon_{j} n_{j},
$$

where, as usual, $c_{j \sigma}^{\dagger}\left(c_{j \sigma}\right)$ denote fermionic creation (annihilation) operators, for site $j$ and spin $\sigma \in\{\uparrow, \downarrow\}$, and $n_{j}=\sum_{\sigma} c_{j \sigma}^{\dagger} c_{j \sigma}$ stands for the particle number operator. 
In this paper we use $t=1$. In addition, we also set the lattice constant and the electronic charge to one. The on-site energies $\epsilon_{i}$ contain the linear potential due to the applied homogeneous electric field $\mathcal{E}$

$$
\epsilon_{j}=\left(j-\frac{1}{2}\right) \mathcal{E} .
$$

The electron-electron interaction present in the physical chain is modelled by the Hubbard Hamiltonian

$$
\mathcal{H}_{\mathrm{U}}=\frac{U}{2} \sum_{i \sigma}\left(n_{i \bar{\sigma}}-\frac{1}{2}\right)\left(n_{i \sigma}-\frac{1}{2}\right)
$$

where $U$ represents the repulsive on-site Coulomb interaction.

The dissipation that is mediated by fermionic heatbath chains is described by individual TB chains attached to each physical site. The corresponding part of the Hamiltonian reads

$$
\begin{aligned}
\mathcal{H}_{\text {bath }}= & -t_{b} \sum_{\alpha j \sigma}\left(d_{\alpha j \sigma}^{\dagger} d_{\alpha+1, j \sigma}+\text { h.c. }\right)+\sum_{\alpha j \sigma} \epsilon_{j} n_{\alpha j \sigma}^{d} \\
& -v \sum_{j \sigma}\left(c_{j \sigma}^{\dagger} d_{1 j \sigma}+\text { h.c. }\right)
\end{aligned}
$$

where $d_{\alpha j \sigma}^{\dagger}$ and $d_{\alpha j \sigma}$ are creation and annihilation operators for particles with spin $\sigma$ at position $\alpha$ within the $\mathrm{j}^{\text {th }}$ bath chain, respectively, and $n_{\alpha j \sigma}^{d}$ is the corresponding number operator. The third term represents the hybridization between the $j^{t h}$ site in the physical chain and the corresponding bath chain. As we will see later it is only the ratio of the two external parameters $t_{B}$ and $v$ that affects our results. Therefore we have used $v=t$ throughout this paper. Note that the thermal bath chains experience the same E-field potential energy $\epsilon_{j}$ as the corresponding physical site. Otherwise the hybridization would violate the gauge invariance as discussed in App. A. The bath chains are considered to be in an equilibrium state infinitely far away from the correlated chain with different chemical potentials $\mu$ given by

$$
\mu_{j}=\epsilon_{j}=\left(j-\frac{1}{2}\right) \mathcal{E} .
$$

In the Keldysh Green's function formalism the current $j_{m n}$ between two adjacent lattice sites $m$ and $n$ for the aforementioned Hamiltonian can be obtained from the Keldysh component of the non-equilibrium Green's function:

$$
j_{m n}=-\frac{t}{2 \pi} \int_{-\infty}^{\infty} d \omega \Re\left\{G_{n m}^{K}(\omega)\right\} .
$$

In the next sections we outline how the Keldysh Green's function for the infinite chain for interacting electrons in a homogeneous electric field can be determined.

\section{B. Cluster Perturbation Theory}

One way to treat properties of interacting many-body systems is Cluster Perturbation Theory (CPT)! $\stackrel{47 / 48}{ }$ For non-interacting particles, CPT always gives the exact result. In the presence of electron-electron interaction CPT is no longer exact; it is a first order perturbation theory in the inter-cluster hopping parameters. The accuracy of CPT increases with the size of the clusters that are treated exactly by numerical means.

For the model at hand, which still has a kind of translational invariance, it suffices to perform an exact diagonalization for one 'central cluster'. The Green's function of the central cluster is then glued together iteratively by CPT to form the infinite system. Possible central clusters are depicted in Fig.2.

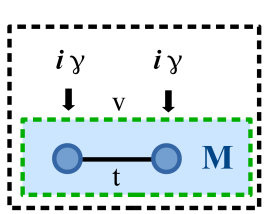

(a)

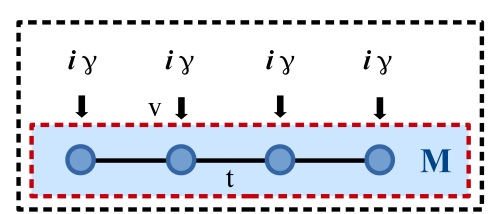

(b)

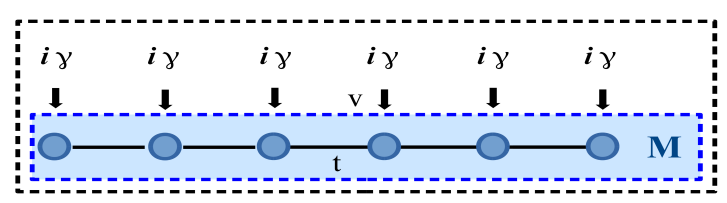

(c)

FIG. 2: (Color online) Central clusters of size $M=2$ (a), $M=$ 4 (b) and $M=6(\mathrm{c})$, which in CPT are periodically strung together to form the infinite physical chain. The dissipation mechanism is represented by the wide-band result $i \gamma$.

\section{Non-equilibrium CPT for the Keldysh Green's functions}

The CPT approach can be generalized to Keldysh Green's Functions. $\stackrel{49}{ }$ In previous studies ${ }^{40 / 41}$ a finite device with interacting electrons has been coupled to infinite leads. Here we will generalize these ideas to infinite Hubbard-chains subjected to a homogeneous electric field.

The single-particle Green's functions in this generalization are represented as four-component matrices in Keldysh space 49

$$
\boldsymbol{g}(\omega)=\left(\begin{array}{cc}
g^{r}(\omega) & g^{k}(\omega) \\
0 & g^{a}(\omega)
\end{array}\right) .
$$

Lower case $g$ denotes a Green's functions of an isolated cluster here, while the labels $r, a$ and $k$ stand for retarded, advanced and Keldysh component, respectively. 
Bold letters are used for the full Keldysh Green's function given in eq. (8). The size of the sub-matrices $g^{r}$, $g^{a}$ and $g^{k}$ is determined by the size of the chosen cluster, as we will see later. Note that this notation applies throughout the whole paper. We will neglect the explicit frequency-dependence of the Green's functions in the following for reasons of readability and will re-introduce it where necessary.

In equilibrium, the Keldysh component is related to the advanced/ retarded components via

$$
g^{k}=\left[g^{r}-g^{a}\right]\left[2 f_{F}(\omega, \mu, T)-1\right],
$$

where $f_{F}(\omega, \mu, T)$ denotes the Fermi function depending on the chemical potential $\mu$ and the temperature $T$.

\section{General scheme of CPT}

The basic step of the theory is the coupling of two initially decoupled isolated parts, which we will label by $\alpha, \beta \in\{1,2\}$. For this system the Green's function is cluster diagonal, i.e. $\boldsymbol{g}^{\alpha \beta}=\delta_{\alpha \beta} \boldsymbol{g}^{\alpha \alpha}$. Now the CPT approximation for the Keldysh Green's functions $\boldsymbol{G}$ of the whole coupled system, written in form of a Dyson equation, reads

$$
\begin{aligned}
\boldsymbol{G}^{\alpha \beta} & =\delta_{\alpha \beta} \boldsymbol{g}^{\alpha \alpha}+\sum_{\bar{\alpha} \in \alpha, \beta} \boldsymbol{g}^{\alpha \alpha} * \boldsymbol{T}^{\alpha \bar{\alpha}} * \boldsymbol{G}^{\bar{\alpha} \beta} \\
\boldsymbol{T}^{\alpha \bar{\alpha}} & =\left(\begin{array}{cc}
T^{\alpha \bar{\alpha}} & 0 \\
0 & T^{\alpha \bar{\alpha}}
\end{array}\right) .
\end{aligned}
$$

where the matrix multiplications have to performed in Keldysh space (see eq. (8)). $T^{\alpha \bar{\alpha}}$ stands for the matrix that contains the parameters for the hopping processes that connect the two clusters $\alpha$ and $\bar{\alpha}$. In the $1 \mathrm{~d}$ case with only two clusters considered here, the only non-zero matrix elements of $T^{\alpha \bar{\alpha}}$ are given by

$$
T_{1, M}^{1,2}=-t=T_{M, 1}^{2,1}
$$

where $M$ denotes the size of the cluster.

We are interested in the sub-matrix of $\boldsymbol{G}$ belonging to the first cluster only, i.e. where $\alpha=\beta=1$. Evaluating eq. 10 leads to

$$
\begin{aligned}
G^{11} & =g^{11}+g^{11} \Gamma^{11} G^{11} \\
\Gamma^{11} & :=T^{12} g^{22} T^{21}
\end{aligned}
$$

A Dyson equation in Keldysh space of the form

$$
G=g+g \Gamma G
$$

can be solved for the advanced/ retarded and Keldysh component separately. For the retarded component we find

$$
\left(G^{r}\right)^{-1}=\left(g^{r}\right)^{-1}-\Gamma^{r}
$$

which has the same form as in equilibrium. Advanced and retarded Green's function are related through $G^{a}=$ $\left(G^{r}\right)^{\dagger}$. For the Keldysh component we obtain a special form of the Kadanoff-Baym equation

$$
G^{k}=G^{r}\left\{\left(g^{r}\right)^{-1} g^{k}\left(g^{a}\right)^{-1}+\Gamma^{k}\right\} G^{a} .
$$

If $g$ belongs to a finite size cluster in equilibrium then the first term in curly brackets can be neglected. Inserting eq. (9) into eq. 14 yields $\left(g^{a}\right)^{-1}-\left(g^{r}\right)^{-1}=2 i 0^{+}$. In the applications in the present paper the second term will originate from semi-infinite TB-chains in the wideband limit. It will therefore contribute a finite value for $\Gamma^{k}$ much larger than $0^{+}$, so that the first term can be ignored.

\section{Coupling to dissipative chains}

Now we begin with the construction of the Keldysh Green's function for the infinite system in an electric field. We start out with the computation of the Keldysh Green's function $\boldsymbol{g}^{c l}(\omega)$ for the $M$-site central cluster based on Exact Diagonalization. In this approximation the initially uncoupled parts are in equilibrium. Therefore it suffices to compute the retarded Green's function.

We now use CPT to couple the central cluster to bath chains which are attached according to Fig.1. We are only interested in Green's functions $\boldsymbol{G}^{c c}$, where both indices $\alpha, \beta$ in eq. 10 belong to the central cluster. In this case eq. 111 reads

$$
\begin{gathered}
\boldsymbol{G}^{c c}=\boldsymbol{g}^{c c}+\boldsymbol{g}^{c c} \boldsymbol{\Gamma} \boldsymbol{G}^{c c} \\
\boldsymbol{\Gamma}:=T^{c b} \boldsymbol{g}^{b b} T^{b c}
\end{gathered}
$$

where $b$ denotes the bath chains, see Fig. 1. The matrix $\boldsymbol{\Gamma}$ describes the dissipation and de-phasing of the transport along the physical chain. Due to the nearest neighbour hopping along the bath chains the sub-matrices $g^{b b, \zeta}$ of $\boldsymbol{g}^{b b}$, where $\zeta$ stands for retarded, advanced or Keldysh, are diagonal, i.e

$$
g^{b b . \zeta}=\operatorname{diag}\left(g_{1}^{b . \zeta}, g_{2}^{b . \zeta}, \ldots, g_{M}^{b . \zeta}\right)
$$

with

$$
g_{j}^{b . \zeta}(\omega)=g^{b . \zeta}\left(\omega-\epsilon_{j}, \mu_{j}\right)
$$

Here $g^{b . \zeta}\left(\omega, \mu_{j}\right)$ is the local Green's function of the semiinfinite bath that is connected to site $j$ of the central cluster at the first contact point. $\epsilon_{j}$ is the on-site energy and $\mu_{j}$ the chemical potential in the $j$-th bath chain. At half filling we have $\mu_{j}=\epsilon_{j}$. The chemical potential only 
enters the Keldysh component. For the retarded part of the semi-infinite TB Green's function we have ${ }^{54}$

$$
g^{b . r}(\omega)=\frac{1}{t_{B}}\left\{\frac{\omega}{2 t_{b}}-i \sqrt{1-\left(\frac{\omega}{2 t_{b}}\right)^{2}}\right\}
$$

with

$$
\Im\left\{g^{b . r}(\omega)\right\} \equiv 0 \quad \text { for } \quad|\omega| \geq 2 t_{B} .
$$

The Keldysh component $g^{b . K}$ follows from eq. (9) as

$$
g^{b . k}\left(\mu_{j}\right)=\left[g^{b . r}-\left(g^{b . r}\right)^{\dagger}\right]\left[2 f_{F}\left(\mu_{j}, T\right)-1\right] .
$$

In the wide-band limit $\frac{37 / 42[43}{\sqrt{3}} t_{b} \gg|\omega|$ we find

$$
g^{b, r}(\omega)=-\frac{i}{t_{b}}
$$

In this case, the self-energy $\boldsymbol{\Gamma}$ entering the Dyson equation (eq. 15p) becomes

$$
\Gamma=i v^{2} /\left|t_{b}\right|:=i \gamma
$$

That means that the dissipation is be described by a constant dissipation parameter $\gamma$. More precisely, the retarded part of the cluster Green's function follows from that of the isolated cluster by

$$
\left(\tilde{G}^{c c . r}\right)^{-1}=\left(\tilde{g}^{c c . r}\right)^{-1}+i \gamma
$$

\section{Construction of the infinite system}

Given the Green's function of the central cluster we can iteratively combine these clusters to form the infinite chain. This can be done best by first determining a right/left semi-infinite 'chain' of these clusters with the coupled bath chains and finally connecting them to end up with the desired infinite structure.

We begin with the construction of the semi-infinite part on the right half of the system. Let $G^{11}(\omega)$ stand

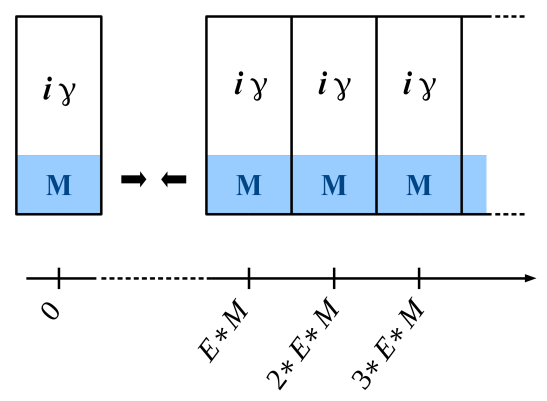

FIG. 3: (Color online) Iterative construction of the right semiinfinite system. The values of the potential energy $\epsilon_{i}$ due to the applied electric field are depicted below (lattice constant and electronic charge are set to unity). for the corresponding Green's function in which the site indices are restricted to the first (leftmost) cluster of the semi-infinite part.

The best way to obtain an iteration equation for this Green's function is given by connecting a dissipative central cluster - with its Green's function $\boldsymbol{G}^{c c}$ - to the first cluster of the remaining semi-infinite part by CPT (see Fig. 3). The coupled system then is the same as the original one apart form an overall energy shift $\omega_{s}=\mathcal{E} M$. Therefore, eq. (11) yields

$$
\boldsymbol{G}^{11}(\omega)=\boldsymbol{G}^{c c}(\omega)\left[1+\boldsymbol{T}^{12} \boldsymbol{G}^{11}\left(\omega-\omega_{s}\right) \boldsymbol{T}^{21} \boldsymbol{G}^{11}(\omega)\right]
$$

This equation can be solved by considering it as an iteration equation starting with $\boldsymbol{G}^{11}(\omega)=\boldsymbol{G}^{c c}(\omega)$. We obtain a similar equation for the left semi-infinite chain with $\omega_{s}=-M \mathcal{E}$. Finally the two semi-infinite chains (clusters) can be combined again by CPT based on eq. 111 (see Fig.4).

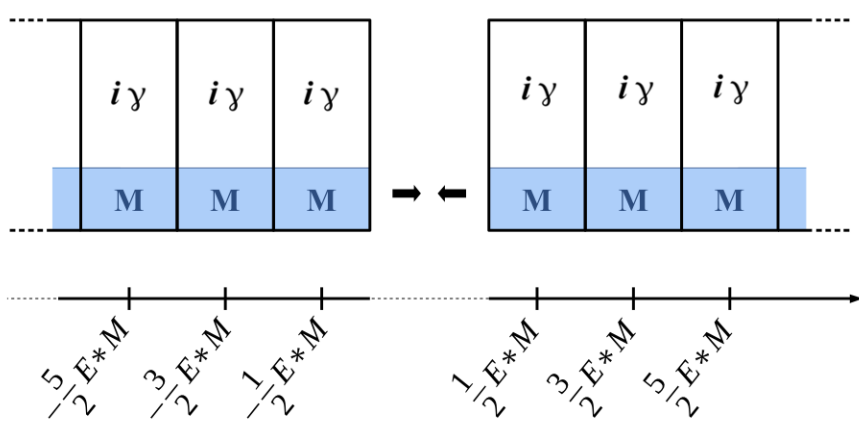

FIG. 4: (Color online) Decomposition of the infinite system into two semi-infinite parts. The zero point of the potential energy is chosen in the middle of the central part.

\section{RESULTS}

\section{A. Non-interacting case}

Now we turn to the results obtained with the Keldysh CPT for the model employed in the present paper. First we present a short summary of the key results in the non-interacting case and compare the description of the dissipative bath chains by semi-infite TB chains to the wide band limit approach.

An instructive approximation is obtained for $|\mathcal{E}|, \gamma \ll$ $|t|$ and wide-band limit, where one finds $\$ 37$

$$
j(\mathcal{E}) \approx \frac{2 \operatorname{taq}}{\pi} \frac{\frac{\mathcal{E}}{2 \gamma}}{1+\left(\frac{\mathcal{E}}{2 \gamma}\right)^{2}} .
$$

Here $q$ is the charge of the particles and $a$ the lattice constant.It should be re-emphasized that we use units in 
which $q=1, a=1, t=1$. This approximate result has a universal maximum, characterized by

$$
\begin{aligned}
& \mathcal{E}_{\text {max }}=2 q a \Gamma, \\
& j_{\text {max }}=\frac{t}{\pi} .
\end{aligned}
$$

As emphasized before, in the non-interacting case CPT provides the exact result, irrespective of the cluster size. Therefore we here also compare our results obtained by the Keldysh CPT approach to previous works. $37 / 42$ And indeed for $U=0$ we find that our CPT results for the infinite non-interacting TB-chain are in perfect agreement with those for the same system obtained in temporal gauge ${ }^{37}$ For non-interacting particles and in the wide-band limit of the bath-chains it turns out that the coupling of the fermionic bath-chains modifies the cluster (advanced) self-energy by a constant damping $\gamma$, as already indicated by eq. (21).

Fig. 5 compares the current $j(\mathcal{E})$ in the physical chain as a function of the electric field $\mathcal{E}$ for the two different approaches for the dissipative bath chains. Curves labeled by the value for the constant damping $\gamma$ correspond to the wide-band limit approach, while the ones where dissipation is described by semi-infinite TB chains are labeled by the value of $t_{B}$. The behaviour of the current is characterized by a pronounced uni-model structure. While peak position and height are reasonably well described for all sets of parameters by the approximation in eq. (24), one observes significant deviations in the current curve for large values of $\gamma$ and small values of $t_{B}$ respectively. However, already for $\gamma=0.1\left(t_{B}=10\right)$ we find an almost perfect quantitative agreement even up to $\mathcal{E}=1$, where the condition $\mathcal{E} \ll t$ is not really met. Since we will restrict our further investigations to the $\gamma<0.1$ regime, the wide-band limit approach chosen in the following is completely justified.

eq. (23) emphasizes the importance of the heat-bath. Without bath chains $(\gamma=0)$ the resulting current would be zero for all values of $\mathcal{E}$, as it was also observed in recent studies for the $1 \mathrm{D}^{37}$ and $2 \mathrm{D}^{38}$ Hubbard model.

The linear relationship $j(\mathcal{E}) \propto \mathcal{E}$ valid for weak electric fields, corresponds to Ohm's Law formulated in the Drude model:

$$
\left.\frac{j}{\mathcal{E}}\right|_{\mathcal{E} \ll \gamma} \approx \frac{t}{\pi \Gamma} \propto \frac{e^{2} \tau}{m^{*}},
$$

where $\tau$ denotes the lifetime of a transport electron with an effective mass $m^{*}$ and a charge $e$ and the TB relationship $m^{*} \propto \frac{1}{t}$ and $\gamma \propto \frac{1}{\tau}$ was used!55[56] The expression essentially represents the well-known Drude DC conductivity per electron.

The initial linear region agrees with that obtained by linear response theory. 57 The occurrence of a maximum value for the current in combination with a subsequent negative differential conductance can be explained by the

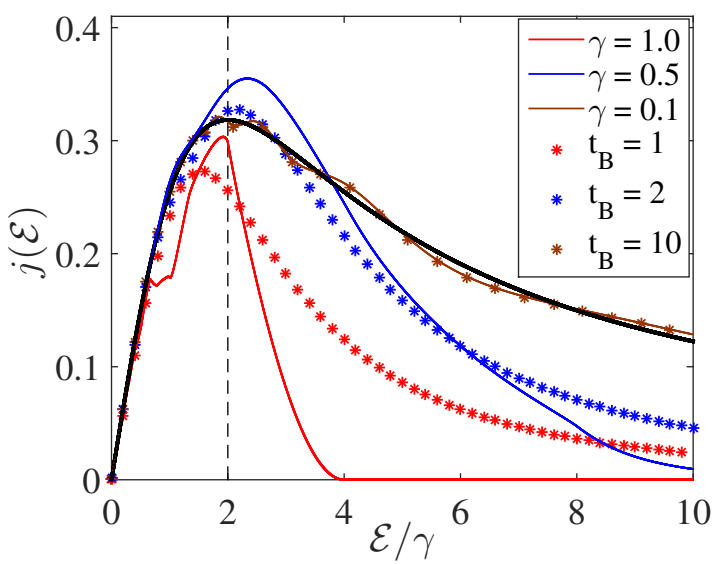

FIG. 5: (Color online) Current calculated according to eq. (7) in the non-interacting case $(U=0)$. Shown with full lines are the results for dissipation described by a wide-band limit approach. In comparison the results for dissipation described by semi-infinite TB chains are shown with star markers. The approximate current gained by evaluating eq. 23 is plotted for comparison (black line). The position of its maximum is marked by a dashed line.

increasing relevance of BOs at larger electric field intensities: When the electron moves to a neighbouring site of lower potential energy, its kinetic energy increases by $\mathcal{E}$ (in the present units). At the same time part of the energy is dissipated into the heat-bath chain. The energy loss due to dissipation is given in terms of the effective damping $\gamma$. For $\mathcal{E} \gg \gamma$ the dissipation mechanism is simply too weak to dissipate enough energy and consequently prevent the occurrence of BOs, which results in a continuously decreasing current. Similar results have been reported recently for the 2D Hubbard model ${ }^{38}$

\section{B. Current in the Hubbard-Wannier-Stark model}

Next we want to study the impact of electronic correlations. For $U \neq 0$ the cluster size $M$ becomes important. We start the following investigations with a two-site cluster $(M=2)$. We will also present the results for larger cluster sizes $(M=4$ and $M=6)$ before turning back to the two-site cluster treatment to explain the origin of the arising current resonances in the Hubbard-Wannier-Stark model.

Fig. 6 shows the dependence of the current $j(\mathcal{E})$ on the interaction strength. In all cases we used a damping parameter $\gamma=0.01$. For $U \leq 0.5$ we find similar $j(\mathcal{E})$-curves as for $U=0$, where the current maximum decreases slightly with increasing $U$. For $U \geq 1$ an oscillatory behaviour sets in and a transport gap opens up. This gap is however much smaller then the single-particle gap that one obtains for $\mathcal{E}=0$ in the density of states.

Similar resonances have been found experimentally in GaAs $/ \mathrm{Al}_{x} \mathrm{Ga}_{1-x}$ As superlattices ${ }^{58}$ The origin of the resonant structures in the current visible in Fig. 6 will be 

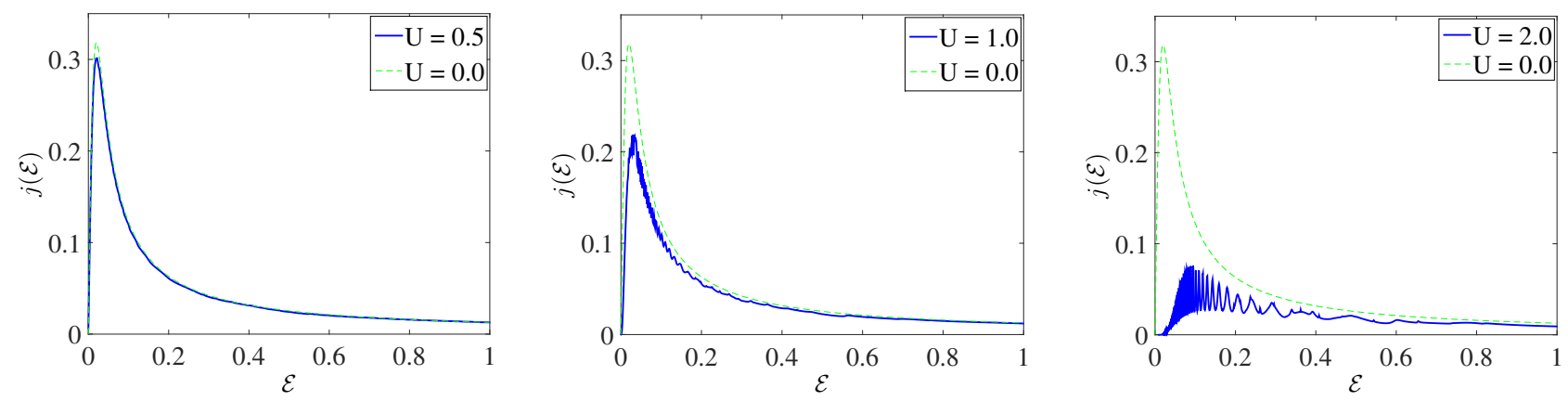

FIG. 6: (Color online) Current $j(\mathcal{E})$ (solid line, calculated according to eq. $(7)$ ) for different values of the interaction strength $U$ $=0.5,1.0,2.0$ and an effective damping $\gamma=0.01$. The $U=0$ result is shown as reference in every subplot (green dashed line).
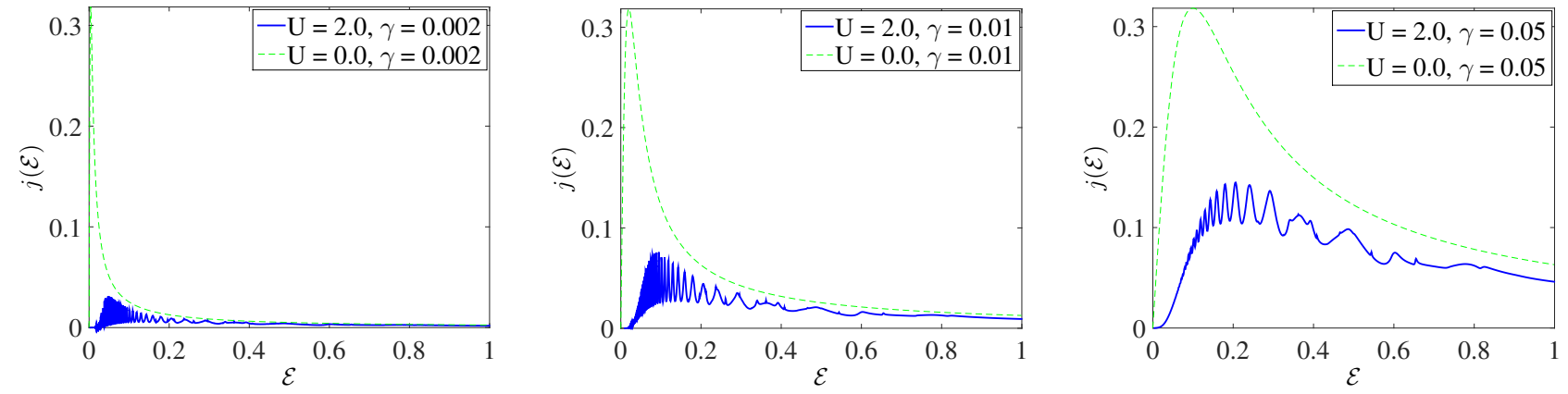

FIG. 7: (Color online) Current $j(\mathcal{E})$ (blue solid line, calculated according to eq. $(7)$ ) for different values of the effective damping $\gamma=0.002,0.01,0.05$ and a fixed value for the repulsive on-site energy $U=2.0$. The corresponding $U=0$-result is shown as reference (green dashed line).

discussed in Sec. IIIC

First, however, we will study the effect of the dissipation strength on the current characteristics, which are displayed in Fig.7 for a fixed value of $U=2.0$ and various values of $\gamma$. There are two major effects observable in Fig.7. First of all, the current maximum $j_{\max }$ increases monotonically with increasing dissipation strength. This is in contrast to the result at $U=0$, where only the position of the maximum current is shifted according to eq. 24). But it corroborates that a non-zero DC current is only possible when dissipation is included. The second effect is a smoothing of the resonances with increasing dissipation strength. While one finds a very spiky structure in the $j(\mathcal{E})$ curve for a small effective damping, the oscillations for larger values of $\gamma$ are rather smooth. The origin of this behaviour will be discussed in the following section.

Now we focus on the influence of larger cluster sizes on the current characteristics analyzed in Fig.8. Independent of the interaction strength the qualitative behavivour does not change significantly when the number of sites within the CPT cluster is increased. The spiky structure of the current remains as well as the finite transport gap. Furthermore, comparing the results for $M=4$ and $M=6$ it seems that in a wide region of the electric field the results coincide also in a quantitative way.
Due to the rather weak impact of the cluster size we will restrict our further investigations to the $M=2$ case.

\section{Origin of the resonances in the Hubbard-Wannier-Stark model}

We will argue in this section that the origin of the resonant structures can be traced back to the occurence of short-range antiferromagnetic order. Although it is well known that the 1D Hubbard model in equilibrium does not exhibit long-range order, $\frac{5960}{5}$ strong short-range fluctuations are nevertheless present. On the short length scale of the cluster, where true long-range and shortrange order can not be distinguished, this order can be described reasonably well by a mean-field decoupling, i.e. by the Stoner model. We will show in the following, that the positions of the resonances are in perfect agreement with the maxima of the eigenvalues of the WSL obtained in this mean-field treatment.

The mean-field decoupled Hamiltonian is given by

$$
\hat{\mathcal{H}}_{1} \approx \hat{\mathcal{H}}_{1}^{\mathrm{MF}}=\frac{U}{2} \sum_{j \sigma}\left\langle\left(n_{j \bar{\sigma}}-\frac{1}{2}\right)\right\rangle\left(n_{j \sigma}-\frac{1}{2}\right),
$$



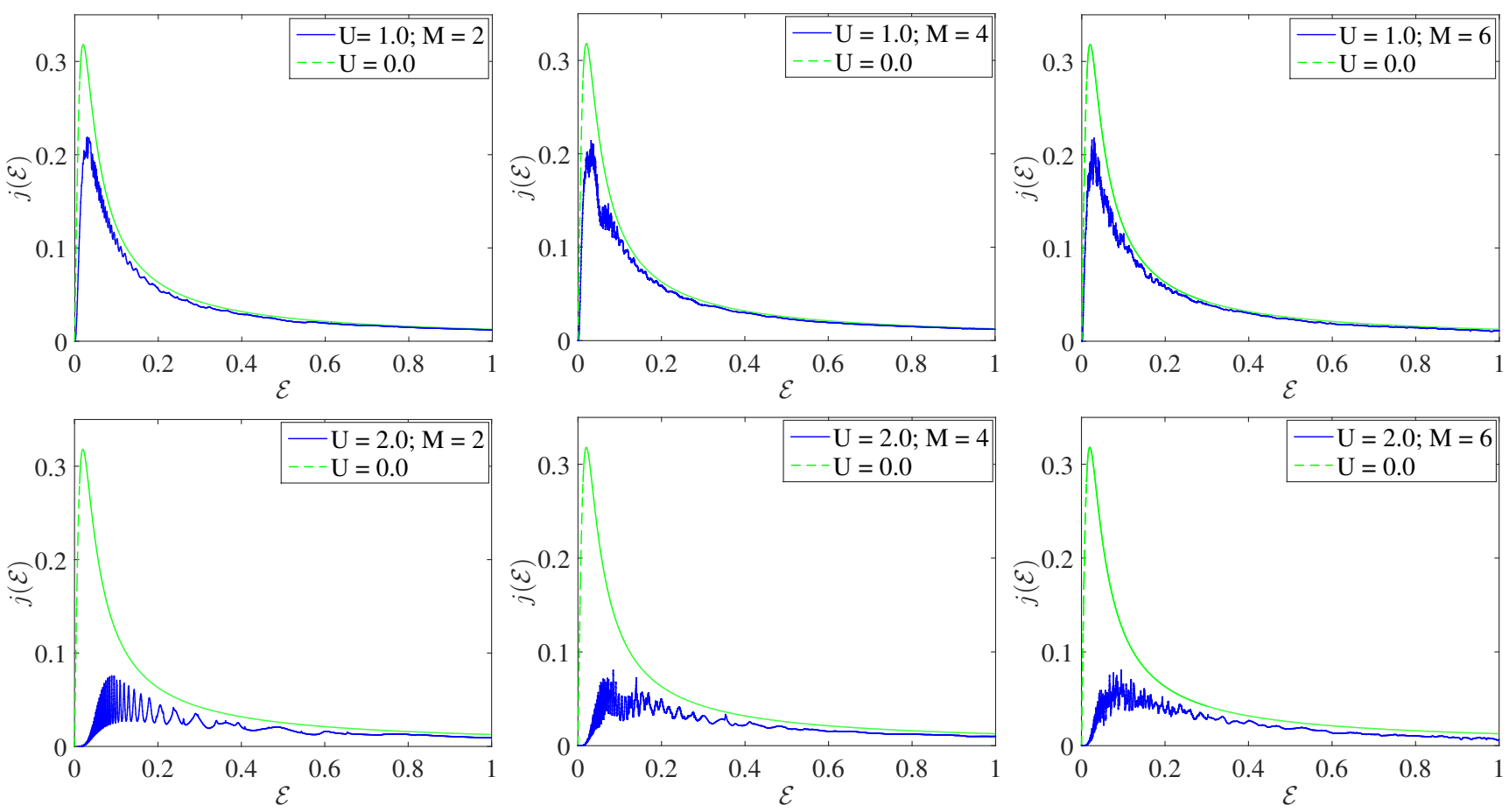

FIG. 8: (Color online) Current $j(\mathcal{E})$ (blue solid line, calculated according to eq. (7)) for different cluster sizes $M=2,4,6$. The results are shown for an interaction strength $U=1.0$ (top row) and $U=2.0$ (bottom row) with a fixed value of the effective damping $\gamma=0.01$. The corresponding $U=0$ result is shown as reference (green dashed line).

Assuming anti-ferromagnetic order leads to

$$
\hat{\mathcal{H}}_{1}^{\mathrm{MF}}=\epsilon \sum_{j \sigma} e^{i \pi j}\left(n_{j \sigma}-\frac{1}{2}\right),
$$

where the value for the order parameter $\epsilon$ will be adjusted such that the Stoner model gives the same singleparticle energy gap as the Hubbard model. The inset of Fig.9 shows the equilibrium Density of States (DOS) $\rho(\omega)$ for the Hubbard and the Stoner model. Obviously, the gap and the low-lying excitations are well described by the Stoner model.

More importantly, we see that the current characteristics of the two models are qualitatively in very good agreement. Concerning the position of the resonances, we see even perfect quantitative agreement. This allows a transparent explanation of the resonances in the frame of the much simpler Stoner model. To this end we start out with the bare Stoner model $\left(\hat{\mathcal{H}}^{\mathrm{WSL}}=\hat{\mathcal{H}}_{0}+\hat{\mathcal{H}}_{1}^{\mathrm{MF}}\right)$ without coupling to the bath chains.

It is well known (see e.g. ref. 21) that non-interacting electrons in a periodic potential, which experience in addition a homogeneous electric field, are trapped in localized states, corresponding to Bloch-oscillators. The energies form Wannier-Stark ladders (WSL). In the present example of an alternating potential the WSL-energies have the form where $\Delta E_{n}$ denotes a possible energy correction. Without the periodic potential $(\epsilon=0)$ the WSL-energies are simply $E_{n}=n \mathcal{E}$, corresponding to $\Delta E_{n}=0$. It is straightforward to determine the eigenvalues (and hence $\left.\Delta E_{n}\right)$ of $\hat{\mathcal{H}}^{\mathrm{WSL}}$ numerically for sufficiently large systems. The result is depicted in Fig. 10 for $\epsilon=0.5$ as function of $\ln (\mathcal{E})$. We observe that for $\mathcal{E} \gg 1$ the energy correction $\Delta E_{n}$ approaches the value $\epsilon$, the amplitude of the alternating potential. For $\mathcal{E}<1$ the energy correction oscillates with extrema at $\mathcal{E}_{\nu}^{*}$ as indicated in Fig.10. Very surprisingly, it turns out that these field strengths correspond to the values of $\mathcal{E}$ where the current maxima are observed in Fig.9. This is illustrated in Fig.11, where the extrema of $\Delta E_{n}$ are compared with the position of the current resonances.

In order to unravel the origin of these oscillations in $\Delta E_{n}(\mathcal{E})$ it is instructive to solve the eigenvalue problem in first order perturbation theory.

The eigenvectors of $\hat{\mathcal{H}}_{0}$ can be expressed as 21

$$
\left|\Psi_{m}\right\rangle=\sum_{n} J_{n-m}(\zeta) c_{n}^{\dagger}|0\rangle
$$

where $J_{l}(\zeta)$ are Bessel functions of the first kind and $\zeta=\frac{2 t}{\mathcal{E}}$. In App. C we show that the first order energy corrections are given by

$$
\Delta E_{m}^{(1)}=\left\langle\Psi_{m}\left|\mathcal{H}_{1}^{\hat{\mathrm{MF}}}\right| \Psi_{m}\right\rangle=e^{i \pi m} \epsilon J_{0}(2 \zeta) .
$$




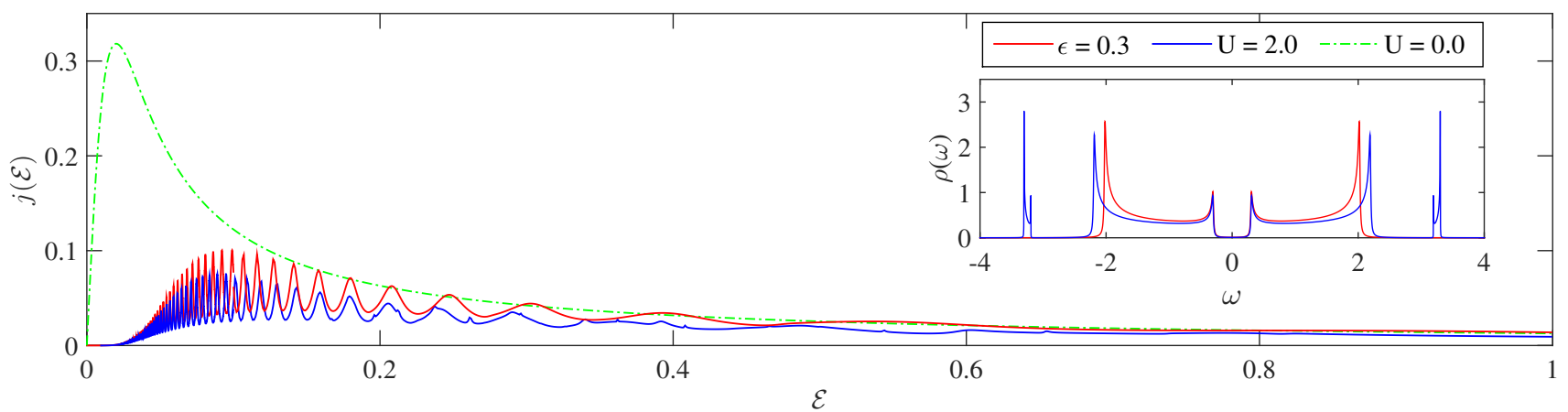

FIG. 9: (Color online) Comparison of the current characteristics $j(\mathcal{E})$ obtained for the Hubbard model and its mean-field approximation, both for $\gamma=0.01$. For the Hubbard model $U=2.0$ and in the mean-field approximation the corresponding order parameter is $\epsilon=0.3$. The latter is adjusted such that both models have the same single-particle energy gap, as can be seen in the inset, which depicts the corresponding equilibrium DOS.

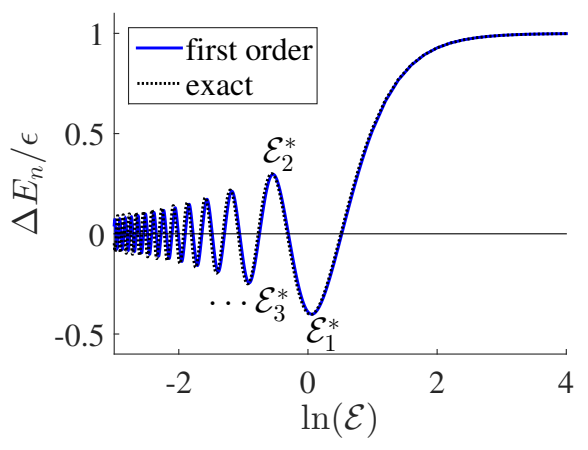

FIG. 10: (Color online) Energy correction $\Delta E_{n}=E_{n}-n \mathcal{E}$ for even $n$ as function of the logarithmic field strength. A comparison is given between the first order correction and the exact value, which is obtained numerically by exact diagonalization. Note that $\Delta E_{n}$ it is the same for all even sites and for odd $n$ the sign is reversed.

The first order correction is also plotted in Fig.10 and compared with the exact correction $\Delta E_{n}:=E_{n}-n \mathcal{E}$. Obviously, the energy correction is well described by the first order term and it also shows the oscillatory dependence on $\mathcal{E}$. To understand this behaviour it is crucial to bear in mind that the Wannier-Stark states $\left|\Psi_{n}\right\rangle$ are localized with mean position $\langle m\rangle=n$ and variance

$$
\sigma^{2}:=\left\langle(m-\langle m\rangle)^{2}\right\rangle=\frac{\zeta^{2}}{2}=\frac{2 t^{2}}{\mathcal{E}^{2}} .
$$

For completeness the proof is given in App. B

Consequently, for fields $\mathcal{E}>\sqrt{2}$ the state is confined to a single site, $n=0$ say, and the first order energy correction is simply $\Delta E_{0}^{(1)}=\epsilon$, corroborating the asymptotic behaviour in Fig. 10 for $\mathcal{E} \gg 1$. Then, upon lowering $\mathcal{E}$ the wavefunction spreads out like $1 / \mathcal{E}$ and reaches gradually sites with alternating potential, which leads to an oscillatory behaviour of $\Delta E^{(1)}$ as observed in Fig. 10 .
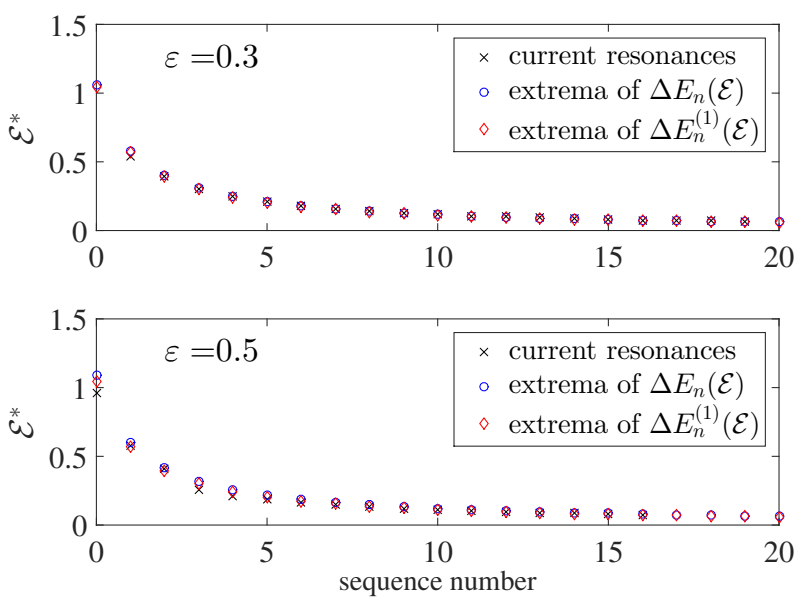

FIG. 11: (Color online) Field values corresponding to the current resonances for the Stoner model, depicted in Fig.9. compared with the positions of extrema of $\Delta E_{n}(\mathcal{E})$ for the exact eigenvalues and those obtained by first order perturbation theory, as discussed in the text.

In perturbation theory, we can also determine the position of the extrema of $\Delta E_{n}^{(1)}(\mathcal{E})$ analytically

$$
\left.\left.\frac{\partial}{\partial \mathcal{E}} \Delta E_{m}^{(1)}(\mathcal{E})\right|_{\mathcal{E}=\mathcal{E}^{*}} \propto \frac{\partial}{\partial \mathcal{E}} J_{0}\left(\frac{4 t}{\mathcal{E}}\right)\right|_{\mathcal{E}=\mathcal{E}^{*}} \propto J_{1}\left(\frac{4 t}{\mathcal{E}^{*}}\right) \stackrel{!}{=} 0
$$

Let $\xi_{\nu}^{(1)}$ be the $\nu$-th zero of $J_{1}(x)$ then

$$
\mathcal{E}^{*}=\frac{4 t}{\xi_{\nu}^{(1)}} \text {. }
$$

Interestingly, to first order, the positions of the maxima are independent of $\epsilon$. In Fig.11 these positions are also included. We find that the first order result is in reasonable agreement with the current resonances. 
We can now invoke second order Fermi's golden rule to compute the current induced by the coupling to the bath chains. The first order terms vanish as the coupling to the bath changes the number of electrons in the physical chain. Brute force application of Fermi's golden rule shows that the energy correction $\Delta E_{n}(\mathcal{E})$ play a decisive role and Fermi's golden rule corroborates the above observation that the current maxima occur at the extrema of $\Delta E_{n}(\mathcal{E})$.

Now that we have shown that the short-range order is responsible for the current resonances, we want to analyze the current suppression for small $\mathcal{E}$ observed in Fig.9.

For small $\mathcal{E}$ the Wannier-Stark states are the wrong starting point. In this case we have to consider $\mathcal{E}$ as perturbation. Then the localized states are due to the alternating potential barrier and have an $\mathcal{E}$-independent localization width. In Fig.12 a sketch of the localized wavefunction centred at some site $r_{1}$ is depicted. The $\mathcal{E}$ field induced linear potential allows the particle to tunnel through the potential barrier that ends at site $r_{2}$. The escape probability is proportional to the size of the filled area in Fig.12.

For a qualitative description we approximate the left half of the wave function by an exponentially decreasing function

$$
\psi(r) \propto \exp \left(-\frac{\left|r-r_{1}\right|}{\xi}\right) .
$$

The quantity $\xi$ describes the spatial extent of the localized wavefunction.

Then the escape probability and in turn the current should be proportional to

$$
j(\mathcal{E}) \propto \int_{-\infty}^{r_{2}}|\psi(r)|^{2} d r \propto \exp \left(-\frac{2\left(r_{1}-r\right)}{\xi}\right) .
$$

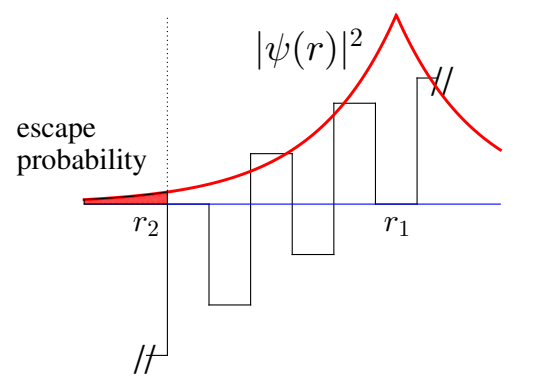

FIG. 12: (Color online) On-site energies (solid black line) of the Stoner model in a homogeneous electric field with antiferromagnetic order parameter. The red curve sketches the wavefunction localized in the potential well at site $r_{1}$. The potential well ends on the left site at position $r_{2}$. The escape probability is proportional to the size of the filled red area.

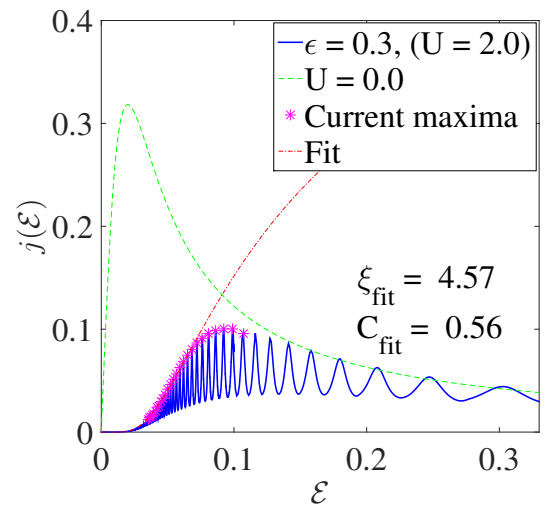

FIG. 13: (Color online) Plot of Fig.9 zoomed into the low electric field region. The maxima are highlighted (magenta star markers) and fitted according to eq. (34) (red dasheddotted lines) resulting in the fit parameters $\xi_{\text {fit }}, C_{\text {fit }}$, depicted in the figures.

The distance $r_{2}-r_{1}$ can be approximated by the slope of the linear potential $r_{2}-r_{1} \approx 2 \epsilon / \mathcal{E}$. Hence, we have

$$
j(\mathcal{E})=C \exp \left(-\frac{4 \epsilon}{\mathcal{E} \cdot \xi}\right) .
$$

We consider $\xi$ as fit parameter, which is estimated from the height of the resonances for small electric fields. The resulting envelope is depicted in Fig.13. The envelope function describes the values of the current maxima almost perfectly for small $\mathcal{E}$, which confirms our description. The size of the transport gap, defined by the field strength $\mathcal{E}^{*}$ for which the exponential in eq. (34) is unity, is characterized by

$$
\mathcal{E}^{*}=\frac{4 \epsilon}{\xi} .
$$

It is proportional to the height potential barrier and inverse proportional to the localization length. For large $\mathcal{E}$ the current is suppressed by the BOs in agreement with the non-interacting result, which is also shown in Fig.13.

\section{Consequences/Relevance of non-local effects in the self-energy}

Our CPT results deviate from a recent investigation of the same system within DMFT $\stackrel{46}{6}$ This can be especially seen in the linear response behaviour: while our calculations suggest the infinite $1 \mathrm{~d}$ chain to be in an insulating state, the DMFT calculations display a metallic linear response. This insulating state with a too large gap is a known drawback of CPT also known from the Hubbard I approximation which corresponds to using a single-site cluster $(M=1)$, as can be seen in Fig. 14 (red curve). This drawback can be corrected by adding an additional uncorrelated bath site in the sense of a minimal DMFT setup. The parameters of the bath site 
can be determined, e.g., within the Variational Cluster Approach (VCA) ${ }^{61}$ Fig. 14a (blue curve) shows the resulting current in the linear response region. Here, it is appropriate to use the parameters determined for the equilibrium case $\mathcal{E}=0$. As one can see, the insulating behaviour has changed to a metallic one. On the other hand, at half filling, the Hubbard model is expected to be insulating for any $U>0$ both in one as well as in two dimensions. ${ }^{64 \mid 65}$ This behavior is clearly missed when neglecting nonlocal effects in the self-energy as in single-site DMFT. A minimal setup to account for the true physical insulating solution down to $U=0$ consists of a two-site cluster $(M=2)$ with additional bath sites, as can be seen from the linear response region displayed in Fig. 14b. These results point out the importance of non-local effects in the self energy, which are induced by short-range antiferromagnetic fluctuations. This is also confirmed in an early VCA work ${ }^{61}$ according to which it is more effective to include physical sites in the cluster, thus making the self-energy more nonlocal, rather than bath sites, which emphasize its dynamical character. This is the reason why we have chosen in this paper to use large clusters rather than smaller ones with bath sites. Nevertheless, it would be interesting in this context to investigate the same infinite $1 \mathrm{~d}$ system also by means of a non-equilibrium cellular DMFT (CDMFT) approach, in particular in a doped situation.
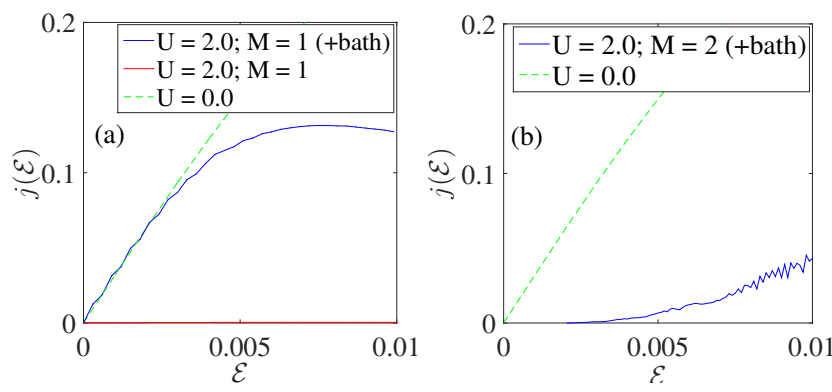

FIG. 14: (Color online) Linear response regime/region of the current $j(\mathcal{E})$ (calculated according to eq. $(7)$ ) with (blue solid line) and without (red solid line) an additional VCA optimized bath site for the single-site case $(M=1)$ (a) and the 2-site case $(M=2)$ (b) for $U=2$. In the single-site treatment the overall linear response behaviour changes from insulating to metallic by adding an additional bath site, while for the $M=2$ case the insulating behaviour remains. All results are shown for a fixed value of the effective damping $\gamma=0.01$. The corresponding result at $U=0$ is shown as reference (green dashed line).

\section{CONCLUSIONS}

We have seen that Hubbard chains in an electric field couped to heat-bath chains exhibit an oscillatory/ resonant behaviour in the current characteristics $j(\mathcal{E})$. The coupling to the heat-bath is essential for a nonvanishing steady-state current. The origin of the oscilla- tions has been traced back to anti-ferromagnetic fluctuations which we mimicked by the Stoner model. Quite generally we have shown that non-interacting electrons in an alternating potential permeated by a homogeneous electric field, i.e. for a Wannier-Stark model with alternating on-site energies, the current exhibits an oscillatory behaviour as function of the field strength $\mathcal{E}$, which is directly linked to similar oscillations in the spacing of the Wannier-Stark ladder. For small field strength the current is suppressed due to localization in the periodic potential barriers. For field strength $\mathcal{E}$ much larger then the damping parameter $\Gamma$, the the current is again suppressed due to localization or rather Bloch-oscillations, which are a ubiquitous feature of the Wannier-Stark model without dissipation.

\section{Acknowledgments}

We acknowledge discussions with Takashi Oka and Fabian Heidrich-Meisner. This work is partially supported by the Austrian Science Fund (FWF) P24081 and P26508. M.A. is supported by the START program of the Austrian Science Fund FWF, project Y746.

\section{Appendix A: Equivalence of the Coulomb and the temporal gauge in the Hubbard-Wannier-Stark model}

Here we will briefly outline a proof, which is closely related to that given in Ref. 66, that in the HubbardWannier-Stark model an electric field can either be described by a potential term, corresponding to the Coulomb gauge used in eq. (1), or in a time-dependent Peierl's phase, corresponding to the temporal gauge. 37 The two approaches are equivalent in the sense that they differ by a unitary transformation which leaves the Liouville equation invariant. As a consequence, the steady state current computed via

$$
j \propto \Im\left\{t_{l+1, l} \cdot \operatorname{tr}\left(\hat{\rho} c_{l+1, \sigma}^{\dagger} c_{l, \sigma}\right)\right\}
$$

will be the same.

To prove this statement, we start out with the Coulomb gauge, where the electric field adds a potential term

$$
\hat{\mathcal{H}}_{\mathcal{E}}=\mathcal{E} \sum_{j} j\left(n_{j}+n_{j}^{b}\right)
$$

to the Hamiltonian $\hat{\mathcal{H}}_{0}$ without $E$-field. The total Hamiltonian is $\hat{\mathcal{H}}=\hat{\mathcal{H}}_{0}+\hat{\mathcal{H}}_{\mathcal{E}}$, where the first term also contains the Hubbard interaction. In the temporal gauge, on the other hand, there is no $\mathcal{E}$-dependent potential term, instead the nearest neighbour hopping parameter $t_{l+1, l}$ is modified to 


$$
t_{l+1, l} \rightarrow \tilde{t}_{l+1, l}=t_{l+1, l} e^{i a q \mathcal{E} t} .
$$

Let the Hamiltonian in temporal gauge be denoted by $\tilde{\mathcal{H}}$. We define a time-dependent unitary operator

$$
U(\tau)=e^{-i \hat{\mathcal{H}}_{\mathcal{E}} \tau}
$$

It can readily be seen that $\tilde{\mathcal{H}}=U^{\dagger} \hat{\mathcal{H}}_{0} U$, where the Hubbard part is not modified, as the unitary operator only contains density operators. Let $\hat{\rho}(t)$ be the density operator defined for the system in Coulomb gauge with the corresponding Liouville equation

$$
\frac{d}{d t} \hat{\rho}=-i[\hat{\mathcal{H}}, \hat{\rho}]
$$
fils

Then the transformed density operator $\tilde{\rho}=U^{\dagger} \rho U$ ful-

$$
\frac{d}{d t} \tilde{\rho}=-i[\tilde{\mathcal{H}}, \tilde{\rho}]
$$

which is the Liouville equation in temporal gauge. The initial value for $t=0$ is the same for both representations, i.e. $\tilde{\rho}_{0}=\hat{\rho}_{0}$. It should be noted that the gauge invariance is violated if the bath chains do not experience the same E-field potential as the corresponding physical site. The reason is the coupling term between the physical sites and the thermal bath. In view of the unitary transformation it is obvious that the current defined in eq. (A1) is the same in both representations, $j_{l+1, l}=\tilde{j}_{l+1, l}$, since

$$
\Im\left\{t_{l+1, l} \cdot \operatorname{tr}\left(\hat{\rho} c_{l+1, \sigma}^{\dagger} c_{l, \sigma}\right)\right\}=\Im\left\{\tilde{t}_{l+1, l} \cdot \operatorname{tr}\left(\tilde{\rho} c_{l+1, \sigma}^{\dagger} c_{l, \sigma}\right)\right\} .
$$

\section{Appendix B: Properties of the WSL wavefunction for a TB model}

Here we outline some of the properties of WSL states of eq. 29 . The key element are the Bessel functions in the following representation

$$
J_{n}(\gamma)=\frac{1}{2 \pi} \int_{-\pi}^{\pi} e^{i(k n-\gamma \sin (k))} d k
$$

The mean position of the WSL state $\left|\Psi_{m}\right\rangle$ is

$$
\begin{aligned}
\langle j\rangle & =\sum_{j} j\left|J_{m-j}(\gamma)\right|^{2} \\
& =m \underbrace{\sum_{l}\left|J_{l}(\gamma)\right|^{2}}_{=1}-\underbrace{\sum_{l} l\left|J_{l}(\gamma)\right|^{2}}_{=0}=m .
\end{aligned}
$$

Similarly for the variance we find

$$
\begin{aligned}
\sigma^{2} & =\left\langle(j-\langle j\rangle)^{2}\right\rangle \\
& =\sum_{l=-\infty}^{\infty} l^{2}\left|J_{l}(\gamma)\right|^{2} \\
& =\frac{1}{(2 \pi)^{2}} \int d k d k^{\prime} e^{i \gamma\left(\sin (k)-\sin \left(k^{\prime}\right)\right)} \sum_{l} l^{2} e^{-i l\left(k-k^{\prime}\right)} \\
& =-\frac{1}{2 \pi} \sum_{k k^{\prime}} e^{i \gamma\left(\sin (k)-\sin \left(k^{\prime}\right)\right)}\left(\frac{\partial}{\partial k}\right)^{2} \delta\left(k-k^{\prime}\right) .
\end{aligned}
$$

Integration by parts eventually yields

$$
\sigma^{2}=\frac{\gamma^{2}}{2}=\frac{2 t^{2}}{\mathcal{E}^{2}}
$$

\section{Appendix C: Perturbation theory for an alternating potential in a Wannier-Stark model}

Here we compute the first order energy correction of $\hat{\mathcal{H}}_{0}+\hat{\mathcal{H}}_{1}^{\mathrm{MF}}$. The terms of the Hamiltonian are defined in eq. (2) and eq. 26.

$$
\begin{aligned}
\Delta E_{m}^{(1)} & =\epsilon \sum_{l}\left|J_{l-m}(\gamma)\right|^{2} e^{i \pi l} \\
& =e^{i \pi m} \epsilon \sum_{n}\left|J_{n}(\gamma)\right|^{2} e^{i \pi n} \\
& =e^{i \pi m} \epsilon \frac{1}{(2 \pi)^{2}} \sum_{k k^{\prime}} e^{i \gamma\left(\sin (k)-\sin \left(k^{\prime}\right)\right)} \sum_{n} e^{i n\left(k-k^{\prime}+\pi\right)} \\
& =\epsilon e^{i \pi m} \frac{1}{2 \pi} \sum_{k} e^{i 2 \gamma \sin (k)} \\
& =\epsilon e^{i \pi m} J_{0}(2 \gamma) .
\end{aligned}
$$

* jakob.neumayer@tugraz.at

1 F. Bloch, Z. Phys. 52, 555 (1928).

2 C. Zener, R. Soc. Lond. A 145, 523 (1934).

${ }^{3}$ G. Wannier, Phys. Rev. 117, 432 (1960).

${ }^{4}$ D. Emin and C. F. Hart, Phys. Rev. B 36, 2530 (1987).
${ }^{5}$ M. Glück, A. R. Kolovsky, H. J. Korsch, and N. Moiseyev, Eur. Phys. J. D 4, 239 (1998).

6 J. Krieger and G. Iafrate, Phys. Rev. B 33, 5494 (1986).

7 G. Nenciu, Rev. Mod. Phys. 63, 91 (1991).

8 A. Bouchard and M. Luban, Phys. Rev. B 52, 5105 (1995). 
9 J. Feldmann, K. Leo, J. Shah, D. Miller, J. Cunningham, T. Meier, G. von Plessen, A. Schulze, P. Thomas, and S. Schmitt-Rink, Phys. Rev. B 46, 7252 (1992).

10 C. Waschke, H. Roskos, R. Schwedler, K. Leo, H. Kurz, and K. Köhler, Phys. Rev. Lett. 70, 3319 (1993).

11 M. Yamaguchi, M. Morifuji, H. Kubo, K. Taniguchi, C. Hamaguchi, C. Gmachl, and E. Gornik, Solid-State Electronics 37, 839 (1994).

12 M. B. Dahan, E. Peik, J. Reichel, Y. Castin, and C. Salomon, Phys. Rev. Lett. 76, 4508 (1996).

13 S. Wilkinson, C. Bharucha, K. Madison, Q. Niu, and M. Raizen, Phys. Rev. Lett. 76, 4512 (1996).

14 B. Anderson and M. Kasevich, Science 282, 1686 (1998).

15 O. Morsch, J. Müller, M. Cristiani, D. Ciampini, and E. Arimondo, Phys. Rev. Lett. 87, 140402 (2001).

${ }^{16}$ W. Hensinger, H. Häffner, A. Browaeys, N. Heckenberg, K. Helmerson, C. McKenzie, G. Milburn, W. Phillips, S. Rolston, H. Rubinsztein-Dunlop, et al., Nature 412, 52 (2001).

17 M.Morifuji, T. Imai, C. Hamaguchi, A. D. Carlo, P. Vogl, G. Böhm, G. Tränkle, and G. Weimann, Phys. Rev. B 65, 233308 (2002).

18 E. Mendez and G. Bastard, Phys. Today 6, 34 (1993).

19 Tarruell Leticia, Greif Daniel, Uehlinger Thomas, Jotzu Gregor, and Esslinger Tilman, Nature 483, 302 (2012), 10.1038 /nature10871.

20 M. Glück, A. Kolovsky, and H. Korsch, Phys. Rep. 366, 103 (2002).

21 T. Hartmann, F. Keck, H. Korsch, and S. Mossmann, New J. Phys. 6, 2 (2004).

${ }^{22}$ T. Oka, R. Arita, and H. Aoki, Phys. Rev. Lett. 91, 066406 (2003)

23 D. Witthaut, M. Werder, S. Mossmann, and H.J.Korsch, Phys. Rev. E 71, 036625 (2005).

24 L. Arrachea, Phys. Rev. B 70, 155407 (2004).

25 A. Di Carlo, P. Vogl, and W. Pötz, Phys. Rev. B 50, 8358 (1994).

26 V. Grecchi, M. Maioli, and A. Sacchetti, J. Phys. A 26, L379 (1993).

27 V. Grecchi, M. Maioli, and A. Sacchetti, Comm. Math. Phys. 159, 605 (1994).

28 V. Grecchi and A. Sacchetti, Ann. Phys. 241, 258 (1995).

${ }^{29}$ V. Grecchi and A. Sacchetti, Phys. Rev. Lett. 78, 4474 (1997).

30 V. Grecchi and A. Sacchetti, Comm. Math. Phys. 185, 359 (1997)

31 V. Grecchi and A. Sacchetti, Comm. Math. Phys. 197, 553 (1998).

32 V. Buslaev and A. Grigis, J. Math. Phys. 39, 2520 (1998).

33 A. Sacchetti (2013), arXiv:1312.6066v1.

34 D. N. Esfahani, L. Covaci, and F. Peeters, Phys. Rev. B 90, 205121 (2014).

${ }^{35}$ F. Heidrich-Meisner, I. González, K. A. Al-Hassanieh, A. E. Feiguin, M. J. Rozenberg, and E. Dagotto, Phys. Rev. B 82, 205110 (2010).

36 M. Eckstein, T. Oka, and P. Werner, Phys. Rev. Lett. 105, 146404 (2010).

37 J. E. Han, Phys. Rev. B 87, 085119 (2013).
38 A. Amaricci, C. Weber, M. Capone, and G. Kotliar, Phys. Rev. B 86, 085110 (2012).

39 Y. Imry and R. Landauer, Rev. Mod. Phys. 71, S306 (1999).

40 M. Knap, W. von der Linden, and E. Arrigoni, Phys. Rev. B 84, 115145 (2011).

41 M. Nuss, C. Heil, M. Ganahl, M. Knap, H. G. Evertz, E. Arrigoni, and W. von der Linden, Phys. Rev. B 86, 245119 (2012).

42 C. Aron, G. Kotliar, and C. Weber, Phys. Rev. Lett. 108, 086401 (2012).

43 J. E. Han and J. Li (2013), arXiv:1304.4269.

44 N. Tsuji, T. Oka, and H. Aoki, Phys. Rev. Lett. 103, 047403 (2009).

45 D. Roy, J. Phys. 20, 025206 (2008).

46 J. Li, J. Aron, G. Kotliar, and J. Han (2014), arXiv:1410.0626v1.

47 S. G. Ovchinnikov and I. S. Sandalov, Phys. C 161, 607 (1989).

48 D. Sénéchal, D. Perez, and D. Plouffe, Phys. Rev. B 66, 075129 (2002).

49 L. V. Keldysh, Sov. Phys. JETP 20, 1018 (1965).

50 L. P. Kadanoff and G. Baym, Quantum Statistical Mechanics: Green's Function Methods in Equilibrium and Nonequilibrium Problems (Addison-Wesley, Redwood City, CA, 1962), ISBN 9780201410464.

51 J. Schwinger, J. Math. Phys. 2, 407 (1961).

${ }^{52}$ H. Haug and A.-P. Jauho, Quantum Kinetics in Transport and Optics of Semiconductors (Springer, Heidelberg, 1998), ISBN 9783540735618.

53 J. Rammer and H. Smith, Rev. Mod. Phys. 58, 323 (1986).

54 E. Economou, Green's Functions in Quantum Physics (Springer, Heidelberg, 2006), ISBN 3540288384.

55 M. Omar, Elementary solid state physics: Principles and applications (Addison-Wesley, Massachusetts, 1975), ISBN 9780201054828.

56 A. Glatz, V. Kozub, and V. Vinokur, Theory of Quantum Transport in Metallic and Hybrid Nanostructures (Springer, Dordrecht, 2006), ISBN 978140204779.

57 R. Kubo, J. Phys. Soc. Jpn. 12, 570 (1957).

58 A. A. Andronov, E. P. Dodin, Y. N. Nozdrin, and D. I. Zinchenko, Phys. Stat. Sol. C 5, 190 (2008).

59 N. D. Mermin and H. Wagner, Phys. Rev. Lett. 17, 1133 (1966)

60 C. Dahnken, M. Aichhorn, W. Hanke, E. Arrigoni, and M. Potthoff, Phys. Rev. B 70, 245110 (2004).

61 M. Potthoff, M. Aichhorn, and C. Dahnken, Phys. Rev. Lett. 91, 206402 (2003).

62 M. Potthoff, Eur. Phys. J. B 32, 429 (2003).

63 M. Potthoff, Eur. Phys. J. B 36, 335 (2003).

${ }^{64}$ G. Kotliar, S. Y. Savrasov, G. Pálsson, and G. Biroli, Phys. Rev. Lett. 87, 186401 (2001).

${ }^{65}$ T. Schäfer, F. Geles, D. Rost, G. Rohringer, E. Arrigoni, K. Held, N. Blümer, M. Aichhorn, and A. Toschi, Phys. Rev. B 91, 125109 (2015).

66 M. Graf and P. Vogl, Phys. Rev. B 51, 4940 (1995). 